\title{
Pancreatic Neuroendocrine Tumors: How Much Surgery is Safe?
}

\author{
Corina Kim-Fuchs ${ }^{1 \#}$, Meret Rudigier ${ }^{1 \#}$, Thomas Malinka ${ }^{1}$, \\ Daniel Candinas ${ }^{1}$, Beat Gloor ${ }^{1}$, Eliane Angst ${ }^{1,2 *}$ \\ ${ }^{1}$ Department of Visceral Surgery and Medicine, Inselspital, University of Bern, Switzerland \\ ${ }^{2}$ Clinic of Surgery and Orthopedics, Cantonal Hospital Schaffhausen, Switzerland \\ "both authors contributed equally
}

Received: December 17, 2015; Accepted: February 20, 2016; Published: March 07, 2016

*Corresponding author: Eliane Angst, Clinic of Surgery and Orthopedics, Cantonal Hospital Schaffhausen, Geissbergstrasse 81, 8208- Schaffhausen, Switzerland, Tel +41 52634 8719; Email: eliane.angst@dkf.unibe.ch

\begin{abstract}
Background: Neuroendocrine tumors of the pancreas (pNET) are rare. Often the patients are asymptomatic for a long time and present late with metastasized disease. Although there are guidelines for the treatment of these tumors, there is no clear consensus whether the resection of liver metastases may be combined with the primary tumor.
\end{abstract}

Method: We retrospectively analyzed the patients operated at our institution between $1 / 2003$ and $12 / 2012$. The patients were analyzed for demographic and clinical data, surgical treatment, tumor size and stage, histology, complications, survival and tumor recurrence over time.

Results: We analyzed 53 patients, 23 females, 30 males. Patients with a one-step surgical approach to pancreas and liver had similar morbidity and mortality compared to patients with disease confined to the pancreas. The primary tumors were smaller in tumors confined to the pancreas. Angioinvasion as well as positive lymph nodes were strongly correlated with synchronous or metachronous liver metastases. Progression free survival was shorter in patients with primary metastasized disease.

Conclusion: The treatment of pNET is challenging. The surgical approach should be tailored to the patient's general condition. Patients benefit from extended and combined resections even in metastasized or locally advanced situations. Combined pancreatic and hepatic surgery may be performed safely.

Keywords: Pancreas; Neuroendocrine tumor; Outcome; Surgery

\section{Introduction}

Neuroendocrine tumors originate from cells of the neuroendocrine system found as single cells or small groups of cells in the mucosa of the gastrointestinal tract. In the pancreas these tumors are divided in functional and non-functional tumors, which is determined by the tumor's production and secretion of neuropeptides and monoamines. These tumors are rare: epidemiological data from Japan and Canada show a prevalence of pancreatic NET (pNET) of 2.23-5.86/100000 [1, $2]$, insulinoma have an incidence of 1-3/million/year, all other entities are even less frequent [3]. Additionally an increase in non-functional tumors was observed in the last 40 years due to improved imaging techniques and clinical awareness [4-6]. Nonfunctional pNETs mainly affect elderly patients in their sixth and seventh decade, whereas the incidence of insulinomas peaks between 40 and 45 years. There is slight predominance for men in non-functional pNETs, in contrast to a female preponderance in insulinomas $[3,7]$.

The WHO classification of these tumors was updated in 2010. The tumors are graded based on the mitotic count and / or Ki67 index. G1 tumors have a mitotic count of $<2 / 10$ high power fields (HPF) and/or a Ki67 index of < 2\%. G2 tumors have a mitotic count of 2-20/10 HPF and/or 3-20\% Ki67 index. G1 and G2 tumors are defined as neuroendocrine tumors (NET). G3 tumors have a mitotic count $>20 / 10 \mathrm{HPF}$ and/or $>20 \%$ Ki67 index and are defined as neuroendocrine cancer (NEC) [7]. Chromogranin A (CgA) and synaptophysin are typically used in the immunohistochemical detection of these tumors [8].

In adenocarcinoma of the pancreas, a stage IV disease with liver metastases is generally not resectable and has a very poor survival. Therefore a palliative concept is appropriate for these patients. In contrast NET and NEC of the pancreas may benefit from extended surgery, including liver resection. In the analysis of the SEER database (Surveillance, Epidemiology, and End Results program of the National Cancer Institute, United States), survival in patients with metastasized disease was 33 months in NET and 5 months in NEC [9]. Nevertheless survival is higher in specialized centers and may depend on the multidisciplinary approach [10].

Furthermore the reduction of tumor load may reduce the local and systemic symptoms. The local symptoms are nonspecific such as abdominal pain and weight loss. The systemic symptoms depend on the hypersecretion of neuropeptides and monoamines. Specifically the carcinoid syndrome is due to the secretion of serotonin and other vasoactive mediators, which result in characteristic episodes of diarrhea, bronchoconstriction, drop of blood pressure and flush. Long-time exposure of the right heart leads to endocardial damage with subsequent valvular 
stenosis and regurgitation, resulting in right-sided heart failure [11-14].

Nevertheless most tumors are asymptomatic, therefore some patients present very late with metastasized disease. Liver metastases are very common and we offered an individualized treatment to these patients. There is no clear consensus whether the resection of liver metastases may be combined with the primary tumor. We analyzed in our case series whether the combined surgery of liver and pancreas is associated with a higher morbidity and mortality.

\section{Material and Method}

In a 10-year period between January 2003 and December 2012 all the patients treated for a pNET/NEC at the Department of Visceral Surgery and Medicine at the Inselspital, University Hospital of Berne were retrospectively analyzed. This study was approved by and registered with the ethical committee of the University of Bern (06-05-12). We included patients with a histologically proven pNET/NEC operated at our institution. 56 patients fulfilled these criteria. Three patients were excluded on the ground of double cancer (adenocarcinoma in the bowel and synchronous pNET), therefore 53 patients entered the final analysis.

These were analyzed for demographic and clinical data, surgical treatment, operation time, tumor size and stage, histology, complications, hospital stay and follow-up (survival, recurrences and metastases) until December 2013. The analysis of demographic data, ASA classification and secondary diagnoses were used to assess the patient's surgical risk. Disease dependent clinical signs and laboratory values, as well as the clinical diagnostics were recorded.

Primary endpoint of this study was the morbidity and mortality in patient with synchronous liver metastases which received a surgical treatment of the pancreas and the liver in a one-step surgical approach compared to patients with only pancreatic surgery. Secondary endpoints were relapse free survival and risk factors for metastases during the follow up.

To analyze our data we defined major and minor complications as follows: major complications were pancreatic fistula, anastomotic leakage, ischemia and hemorrhage. Minor complications were urinary infection, pneumonia, wound infections, ascites, delayed oral feeding and cardiac complications. Tumors were classified according to the 2010 WHO criteria. TNM staging was performed by pathologists at our institution, following the consensus guidelines elaborated by ENETS (European Neuroendocrine Tumor Society).

The surgical technique varied according to the tumor location and extent. General anesthesia combined in most cases with peridural anesthesia was used together with a transverse laparotomy. Antibiotic prophylaxis and octreotides were given. Pancreatic tail resections were spleen-preserving for well differentiated small tumors. For larger or less differentiated tumors the spleen was resected en bloc with the pancreas.
The Whipple procedure included a duodenopancreatectomy, cholecystectomy, pancreatico-jejunostomy, hepaticojejunostomy, duodeno-jejunostomy in pp-Whipple and Roux-Y-reconstruction in classical Whipple. In case of tumor invasion of portal vein or superior mesenteric vein, these were resected and reconstructed anatomically (3 cases). Oncological lymphadenectomy was always performed except for enucleated insulinomas. The liver metastases were treated by synchronous resection in 9 cases according to the surgeon's judgment.

Postoperative treatment was with early enteral feeding, octreotides for 5 days after pancreatic procedures, thromboembolic prophylaxis with low molecular weight heparin and early mobilization.

\section{Statistical analysis}

Continuous data are presented as mean \pm one standard deviation and a t-test was used where appropriate. Proportions were compared with Chi2 or Fisher's exact test and survival was tested by log rank. An alpha value below 0.05 was determined as significant. All statistics were done with the statistical software (SPSS Statistics version 21, Chicago, Illinois, USA).

\section{Results}

In this retrospective study we included 53 patients. We operated 39 patients without synchronous liver metastases (group I) and 14 patients with synchronous liver metastases at time of diagnosis (group II). For some analysis we divided the groups further in subgroups. Patients in subgroup Ia developed liver metastases over time ( $\mathrm{n}=9)$, patients in subgroup Ib were free on any tumor recurrence over time $(n=16)$ and patients in subgroup Ic had insulinomas $(\mathrm{n}=14)$. Patients in subgroups IIa had a one-step surgical procedure of pancreas and liver $(n=9)$, patients in subgroup IIb had a two-step surgical approach, pancreas first $(n=2)$ and in subgroup IIc only the pancreas was operated on $(n=3)$.

Patient characteristics of the 53 patients are detailed in table 1 . Of these $23(43.4 \%)$ were female and 30 (56.6\%) were male. Mean age at time of diagnosis was $55.8 \pm 12.6$ years. The distribution of the patients' general health was not statistically different. Most patients had additional diagnoses besides the pNET/NEC: $61.5 \%$ of the patients in group I and $78.6 \%$ of the patients in group II. 22 patients (41.5\%) showed clinical signs associated with the tumor (table 2). Preoperative CgA was measured in 24 patients and was increased in 14 (10/14 group I and 4/10 group II).

Preoperative radiological studies were done in all the patients. A computer tomography was performed in 30 patients (76.9\%) of group I with a pathological result in $96.7 \%$ and in all patients of group II with $100 \%$ pathological results. MRI $(n=26)$, ultrasound $(\mathrm{n}=23)$, PET-CT $(\mathrm{n}=10)$ and somatostatin-receptor scintigraphy $(n=17)$ were pathological in $37.7,32.1,15.1$ and $18.9 \%$ of the cases.

All patients were treated surgically. A Whipple procedure was 
Table 1: Patient demographics and treatment.

\begin{tabular}{|c|c|c|c|}
\hline & Group I & Group II & Total \\
\hline $\mathrm{n}=$ & 39 & 14 & 53 \\
\hline $\operatorname{Sex}(f: m)$ & $19: 20$ & $04: 10$ & $23: 30$ \\
\hline Age, yrs (+/- SD) & $56.5(13.8)$ & $54.1(8.6)$ & $55.8(12.6)$ \\
\hline Age, yrs (range) & $31-82$ & $41-70$ & $31-82$ \\
\hline \multicolumn{4}{|c|}{ American Society of Anesthesiologists classification } \\
\hline ASA I: healthy & $3(7.7)$ & $2(14.3)$ & $5(9.4)$ \\
\hline ASA II: mild systemic disease & $21(53.8)$ & $7(50.0)$ & $28(52.8)$ \\
\hline ASA III: severe systemic disease & $11(28.2)$ & $5(35.7)$ & $16(30.2)$ \\
\hline ASA IV: severe life-threatening systemic disease & $4(10.3)$ & 0 & $4(7.5)$ \\
\hline \multicolumn{4}{|l|}{ Other relevant diagnosis } \\
\hline Other diagnosis & $24(61.5)$ & $11(78.6)$ & $35(66.0)$ \\
\hline No other diagnosis & $15(38.5)$ & $3(21.4)$ & $18(34.0)$ \\
\hline \multicolumn{4}{|l|}{ Surgical treatment } \\
\hline \multicolumn{4}{|l|}{ Resection pancreas } \\
\hline Whipple procedure & $17(43.6)$ & $6(42.9)$ & $23(43.4)$ \\
\hline Left resection & $12(30.8)$ & $8(57.1)$ & $20(37.7)$ \\
\hline Others & $10(25.6)$ & 0.0 & $10(18.9)$ \\
\hline Liver surgery & 0 & $9(64.3)$ & $9(17)$ \\
\hline Operation time : minutes (+/-SD) & $350.6(118.6)$ & $392.9(123.9)$ & $361.0(120.2)$ \\
\hline Operation time; minutes (range) & $180-620$ & $230-435$ & $180-620$ \\
\hline Hospital stay: days (+/- SD) & $22.9(19.9)$ & $24.6(12.5)$ & $23.4(18.2)$ \\
\hline Hospital stay: days (range) & $7-128$ & $7-44$ & $7-128$ \\
\hline \multicolumn{4}{|l|}{ TNM } \\
\hline pT1 NO MO:StageI & $9(23.0)$ & 0 & $9(17.0)$ \\
\hline pT2 NO MO:StageIIA & $5(12.8)$ & 0 & $5(9.4)$ \\
\hline pT3 NO MO:StageIIB & $2(5.1)$ & 0 & $2(3.8)$ \\
\hline pT4 NO MO: StageIIIA & $1(2.6)$ & 0 & $1(1.9)$ \\
\hline pT1 N1 MO:StageIIIB & $1(2.6)$ & 0 & $1(1.9)$ \\
\hline pT2 N1 MO:StageIIIB & $2(5.1)$ & 0 & $2(3.8)$ \\
\hline pT3 N1 MO:StageIIIB & $1(2.6)$ & 0 & $1(1.9)$ \\
\hline pT4 N1 MO:StageIIIB & $4(10.3)$ & 0 & $4(7.5)$ \\
\hline pT4 NO M1:Stage IV & 0 & $2(14.3)$ & $2(3.8)$ \\
\hline pT2 N1 M1:StageIV & 0 & $4(28.6)$ & $4(7.5)$ \\
\hline pT2 N1 M1: Stage IV & 0 & $7(50.0)$ & $7(13.2)$ \\
\hline pT3 N1 M1: Stage IV & 0 & $1(7.1)$ & $1(1.9)$ \\
\hline Insulinoma & $14(35.9)$ & 0 & $14(26.4)$ \\
\hline \multicolumn{4}{|l|}{ Complications } \\
\hline Major complications & $6(15.4)$ & $6(42.9)$ & $12(22.6)$ \\
\hline Minor complications & $23(59.0)$ & $10(71.4)$ & $33(62.3)$ \\
\hline
\end{tabular}


Table 2: Blood changes, clinical signs and radiological studies.

\begin{tabular}{|c|c|c|c|}
\hline Blood changes & Group I & Group II & Total \\
\hline Hypoglycemia & $13 / 39$ & $1 / 14$ & $14 / 53$ \\
\hline Hypopotassemia & $1 / 39$ & $1 / 14$ & $2 / 53$ \\
\hline Gastrin increased & $1 / 2$ & $1 / 3$ & $2 / 5$ \\
\hline 5-hydroxyindole acetic acid & 0 & $1 / 4$ & $1 / 4$ \\
\hline Insulin increased & $12 / 14$ & $1 / 2$ & $13 / 16$ \\
\hline VIP & $2 / 3$ & 0 & $2 / 3$ \\
\hline Chromogranin A increased & $10 / 14$ & $4 / 10$ & $14 / 24$ \\
\hline \multicolumn{4}{|l|}{ Clinical signs } \\
\hline Diarrhea & $4(10.3)$ & $3(21.4)$ & $7(13.2)$ \\
\hline Cholelithiasis & $4(10.3)$ & $2(14.3)$ & $6(11.3)$ \\
\hline Dermatosis & $3(7.7)$ & $1(7.1)$ & $4(7.5)$ \\
\hline Steatontioe & $1(2.6)$ & $1(7.1)$ & $2(3.8)$ \\
\hline Flush & $1(2.6)$ & $1(7.1)$ & $2(3.8)$ \\
\hline No weight loss & $27(69.2)$ & $10(71.4)$ & $37(70.0)$ \\
\hline$<2 \mathrm{~kg}$ & $1(2.6)$ & $1(7.1)$ & $2(3.8)$ \\
\hline $2-5 \mathrm{~kg}$ & $5(12.8)$ & $1(7.1)$ & $6(11.3)$ \\
\hline $5-10 \mathrm{~kg}$ & $1(2.6)$ & $1(7.1)$ & $2(3.8)$ \\
\hline$>10 \mathrm{~kg}$ & $5(12.8)$ & $1(7.1)$ & $6(11.3)$ \\
\hline \multicolumn{4}{|l|}{ Radiological examinations } \\
\hline СT & $30(76.9)$ & $14(100)$ & $44(83.0)$ \\
\hline Pathological result & $29(96.7)$ & $14(100)$ & $43(81.1)$ \\
\hline MR1 & $22(56.4)$ & $4(28.6)$ & $26(49.1)$ \\
\hline Pathological result & $17(77.3)$ & $3(75.0)$ & $20(37.7)$ \\
\hline Ultrasound & $17(43.6)$ & $6(42.9)$ & $23(43.4)$ \\
\hline Pathological result & $12(70.6)$ & $5(83.3)$ & $17(32.1)$ \\
\hline PET & $6(15.4)$ & $4(28.6)$ & $10(18.9)$ \\
\hline Pathological result & $4(66.7)$ & $4(100)$ & $8(15.1)$ \\
\hline Somatostatin-receptor scintigraphy & $9(23.1)$ & $8(57.1)$ & $17(32.1)$ \\
\hline Pathological result & $5(55.6)$ & $5(62.5)$ & $10(18.9)$ \\
\hline
\end{tabular}

The blood changes in number of patient/totally measured patients. Clinical signs and radiological studies: number of affected patients and the percentage in brackets.

performed in $43.6 \%$ of the cases in group I, $42.9 \%$ in group II. Left resections were performed in $30.8 \%$ in group I, $57.1 \%$ in group II, other procedures (central resection, total pancreatectomy, enucleation) were performed in $25.6 \%$ in group I and none in group II. The operation time in group I was not shorter than in group II: $350 \pm 118 \mathrm{~min}$ vs. $392 \pm 123 \mathrm{~min}(\mathrm{p}=0.34)$. Likewise, the subgroup analysis showed no longer operation time for subgroup IIa with one-step surgical approach to pancreas and liver $420 \pm 141 \mathrm{~min}$ compared to subgroups IIb/c $344 \pm 72.7 \mathrm{~min}$ $(\mathrm{p}=0.28)$ and group I $(\mathrm{p}=0.17)$ where only the pancreas was resected.

We found a similar length of hospital stay in both groups.
Patients from group I stayed for $22.9 \pm 19.9$ days, patients from group II for $24.6 \pm 12.5$ days ( $p=0.76$ ). There was a trend to less major complications in group I compared to group II $(p=0.06)$. In fact by analyzing the subgroups of group II we found that all patients in subgroups IIb and IIc with liver metastases and no liver surgery, had major complications and only one patient from group IIa with the one-step surgical approach. Comparing group I with subgroup IIa no difference was detected 6/39 (15.4\%) vs. $1 / 9(11.1 \%)(p=1.00)$. There was no difference in minor complications between the groups $(\mathrm{p}=0.68)$. Over all group I and subgroup IIa had the same morbidity and no 30 days mortality (table 1, figure 1). 
Primary tumors in group I were significantly smaller in diameter than tumors in group II ( $30.1 \pm 28 \mathrm{~mm}$ vs. $59.9 \pm 35.1 \mathrm{~mm}$ ) $(p=0.002)$ (figure 2). Tumors in subgroup Ia tended to be larger than in subgroup Ib $(45.6 \pm 22.8 \mathrm{~mm}$ vs. $27.2 \pm 27.8 \mathrm{~mm})(\mathrm{p}=0.09)$. Histologically there was no difference between the two groups in terms of NET (G1-G2) (group I: 20/39 (51\%) vs group II: 7/14 $(50 \%))$. NEC (G3) tended to be less frequent in group I than group II $(1 / 39(2.6 \%)$ vs $3 / 14(21 \%))(p=0.05)$. Insulinomas were only found in group I (14/39 (35.9\%)) (figure 3). Angioinvasion of the primary tumor was correlated with synchronous or metachronous liver metastasis. All patients with synchronous or metachronous liver metastasis had angioinvasion, whereas in the subgroups Ib only $6 / 16$ had an angioinvasion $(p<0.001)$. Patients with R1 (pancreas $\mathrm{n}=2$, liver $\mathrm{n}=2$ ) or $\mathrm{R} 2$ (liver $\mathrm{n}=1$ ) resection had tumor progression within $0-9$ months. In 53 resections 281 lymph nodes were retrieved (median=18.3; range 1 to 37). Patients with positive lymph nodes had a higher risk for metastasis (group I) or recurrence of metastasis (group IIa) ( $p=0.015)$.

Progression free survival was significantly different in group Ia compared to group IIa ( $21 \pm 15.7$ vs. $7.6 \pm 7.9$ months $)(p<0.001)$. In group I 9 patients developed metastases (liver $n=6$, others $n=3)$. The liver metastases were treated by surgery $(n=3)$ and/ or chemotherapy, DOTATOC, radiation and chemoembolization. Three patients died at 47.6 months (31-71), one was lost to

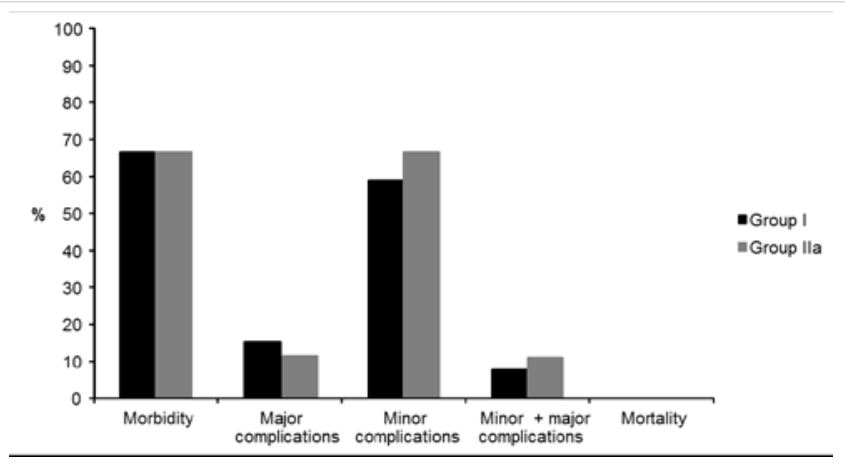

Figure 1: Morbidity and mortality. Percentage of affected patients in group I and IIa are shown for overall morbidity, major and minor complications, patients with both major and minor complications and mortality. There is no difference in morbidity and mortality between these groups.

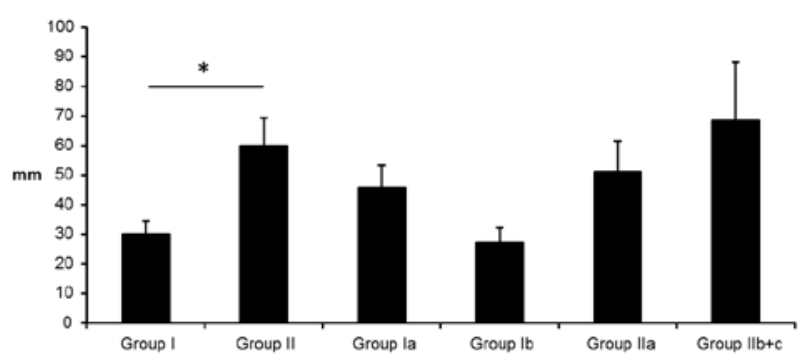

Figure 2: Primary tumor size. Tumor size was measured in $\mathrm{mm}$. Patients from group I had significantly smaller tumors than patients from group II $(30.1 \pm 28.0 \mathrm{~mm}$ vs. $59.9 \pm 35.1)(\mathrm{p}=0.002)$.

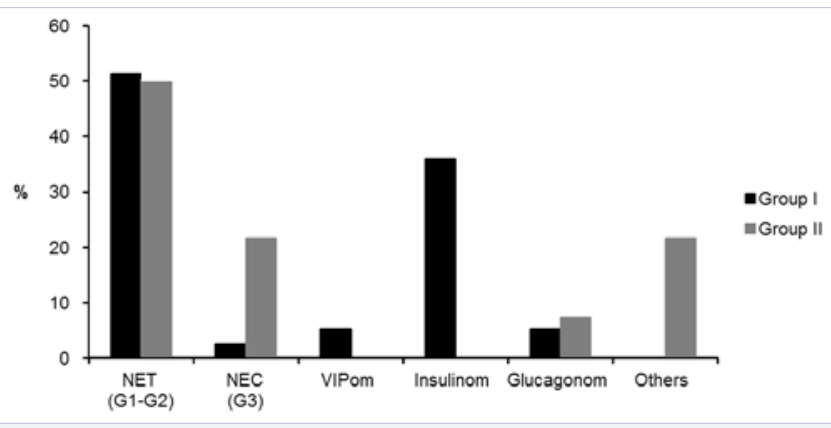

Figure 3: Histology. NETs, NECs, VIPoma and Glucagonoma were found. Results are presented in percentage. Insulinomas were only found in group I (35.9\%). No difference was found between the two groups in terms of NET and a trend for more NEC in group II $(\mathrm{p}=0.05)$.

follow-up at 83 months (table 3). After an R0 resection in group II new metastases occurred after 11.5 months (2 - 22) in 5/5 patients. These were treated by a second liver surgery $(n=4)$ and/or the above mentioned treatments and sandostatin, SIRT or RITA. Orthotopic liver transplantation was performed once, after 17 months of stable follow-up. However this patient developed a pancreatic recurrence and new liver metastases. Four patients died after 33.8 months ( 9 - 83), one was lost to follow-up at 84 months. In group IIb/c one patient died after three month and one was lost to follow-up after 63 months.

\section{Discussion}

The guidelines established by ENETS help to decide on the treatment strategy for neuroendocrine tumors of the pancreas [15]. Nevertheless the question whether liver metastases should be resected and when is still matter of debate and the surgeon's experience. We retrospectively analyzed a cohort of 53 patients operated at our institution to determine whether combined surgery of the pancreas and the liver in metastasized neuroendocrine pancreatic tumors is safe. We compared them to patients operated solely on the pancreas. We found no difference in morbidity and mortality between the two groups.

To prepare for surgery, the preoperative radiological studies are crucial in the decision of the oncological concept. Nevertheless the imaging modalities evolved in the time of the study and there are no definite guidelines for a standard imaging pathway. It depends on tumor differentiation and expression of receptors. Imaging of the pancreatic lesion may be best performed by either endoscopic ultrasound, contrast enhanced CT Scan or MRI ${ }^{7}$. Somatostatin-receptor scintigraphy plays an important role in tumor staging, but is progressively replaced by PET-CT. This development is reflected in the imaging modalities the patients received. No established pathway was used, rather imaging was adapted to the local situation in the pancreas and the need for systemic staging. As recommended in the guidelines, blood testing for hormonal changes was performed upon clinical suspicion.

The decision making about the extent of the resection was discussed on a multidisciplinary board and depended on the 
Table 3: Follow up with or without tumor progression, analyzing death, closure of case and survival. Time in months with range in brackets.

\begin{tabular}{|c|c|c|c|c|c|c|c|c|}
\hline & \multicolumn{2}{|c|}{ Group Ia / b $(n=25)$} & \multicolumn{2}{|c|}{ Group lc $(n=14)$} & \multicolumn{2}{|c|}{ Group Ila $(n=9)$} & \multicolumn{2}{|c|}{ Group Ilb/c $(n=5)$} \\
\hline & $\mathbf{n}$ & Months (range) & $\mathbf{n}$ & $\begin{array}{l}\text { Months } \\
\text { (range) }\end{array}$ & $\mathbf{n}$ & Months (range) & $\mathbf{n}$ & Months (range) \\
\hline Metastases/Recurrences & 9 & $21.0(3-56)$ & 0 & 0 & 9 & $7.6(2-22)$ & - & - \\
\hline Death & 3 & $47.6(31-71)$ & 0 & 0 & 4 & $33.8(9-83)$ & 1 & 3 \\
\hline Lost to follow-up & 1 & 83 & 0 & 0 & 1 & 84 & 1 & 63 \\
\hline No metastases & 16 & - & 14 & - & 0 & 0 & 0 & 0 \\
\hline Death & 1 & 91 & 0 & 0 & 0 & 0 & 0 & 0 \\
\hline Closure of case & 1 & 3 & 14 & $22.8(2-71)$ & 0 & 0 & 0 & 0 \\
\hline Alive & 14 & $45.6(10-91)$ & 0 & 0 & 0 & 0 & 0 & 0 \\
\hline
\end{tabular}

disease load and distribution in the liver, local resectability criteria, age and fitness of the patient as well as intraoperative findings. Oncological lymph node dissection was always performed except for insulinomas. It is a matter of debate whether small tumors may be enucleated or observed. Retrospective analysis with examination of tumor grade and tumor size give different cut-offs. Toste et al. from UCLA found that non-functional tumors below two centimeters may be operated by a tissue sparing technique, although this group found positive lymph nodes in $7.4 \%$ of the cases [16]. In contrast Sharpe et al found that surgical resection of localized pNETs up to two $\mathrm{cm}$ had an overall survival advantage compared to patients, which were observed [17]. Another difficulty in the decision process is the reliability of preoperative histology. A recent study analyzed 58 pNETs and compared the grading of FNA to the resected specimen. The authors found that only if the FNA sample showed adequate cellularity, there was a good correlation of the highest grading in the FNA with the grading in the resected specimen [18]. Often tumor grade is not certain preoperatively in large tumors and in small tumors no biopsies are taken. As for G3 tumors the guidelines recommend resection of the liver only in selected cases [15], this cannot always be decided on the ground of a sound histology but may rely on the experience of the treating team.

Furthermore the extent of lymph node dissection is debatable. Two recent studies using the SEER tumor registry concluded that lymph node dissection is necessary, to predict the outcome of the patient. Furthermore high grade tumors and large tumors predicted nodal positivity and decreased disease specific survival [19-20]. Our findings support this data: patients with positive lymph nodes had a significantly higher risk for metastases or recurrences. Furthermore metastasized tumors were significantly larger. Additionally we found that angioinvasion was correlated with synchronous or metachronous liver metastases $(p<0.001)$. There was a trend for tumor grade to be higher in more aggressive tumors. Also R1 or R2 resection was a predictor of fast recurrence. These data were not statistically significant because of the small sample size in the subgroups. But they are in accord with the published literature.
Operation times and hospital stay were not different due to the small sample size and the inequality of the cases. $\operatorname{CgA}$ measurements were not performed in all the patients, especially in the first years of the series. Jilesen et. Al. showed that in nonfunctional resectable pNETs the diagnostic accuracy of $\operatorname{CgA}$ was moderate, but it is the best marker we may determine [21].

In the analysis of the SEER database, survival in patients with metastasized disease was 33 months in pNET and 5 months in pNEC [9]. Nevertheless survival is higher in specialized centers and may depend on the multidisciplinary approach [10]. Another group showed that the resection of the primary tumor improves patient outcome in non-functional pNET [22]. Despite advanced tumor stages we found relatively long disease free survivals of 21 months in group Ia and 8.7 months in group IIa. Patients with disease recurrence still survived. This may be due to the diverse therapeutic options and the resection of the primary tumor. Surgery adapted to localization, type of tumor and the patient's general condition, is the most important therapeutic approach for localized pancreatic disease, as it significantly improves survival, if based on oncological principles [27, 7]. Through the reduction of the tumor mass, improvement of quality of life and prolonged survival is achieved in advanced disease [7, 15, 23-25].

\section{Conclusion}

The treatment of pNET/NEC is challenging. As often the primary tumors are large and metastasized at the time of diagnosis, the surgical approach should be tailored to the patient's general condition. In our experience patients benefit from extended and combined resections even in metastasized or locally advanced situations. Combined pancreatic and hepatic surgery may be performed safely.

\section{References}

1. Ito T, Sasano H, Tanaka M, Osamura RY, Sasaki I, Kimura W et al. Epidemiological study of gastroenteropancreatic neuroendocrine tumors in Japan. J Gastroenterol. 2010;45(2):234-243. doi: 10.1007/ s00535-009-0194-8.

2. Hallet J, Law CH, Cukier M, Saskin R, Liu N, Singh S. Exploring the rising 
incidence of neuroendocrine tumors: a population-based analysis of epidemiology, metastatic presentation, and outcomes. Cancer. 2015;121(4):589-597. doi: 10.1002/cncr.29099.

3. Jensen RT, Cadiot G, Brandi ML, de Herder WW, Kaltsas G, Komminoth P, Scoazec JY et al. ENETS Consensus Guidelines for the management of patients with digestive neuroendocrine neoplasms: functional pancreatic endocrine tumor syndromes. Neuroendocrinology. 2012;95(2):98-119. doi: 10.1159/000335591.

4. Garcia-Carbonero R, Capdevila J, Crespo-Herrero G, Diaz-Perez JA, Martinez Del Prado MP, Alonso Orduna V, et al. Incidence, patterns of care and prognostic factors for outcome of gastroenteropancreatic neuroendocrine tumors (GEP-NETs): results from the National Cancer Registry of Spain (RGETNE). Ann Oncol. 2010;21(9): 1794-1803. doi: 10.1093/annonc/mdq022.

5. Schimmack S, Svejda B, Lawrence B, Kidd M, Modlin IM. The diversity and commonalities of gastroenteropancreatic neuroendocrine tumors. Langenbecks Arch Surg. 2011; 396(3):273-298. doi: 10.1007/ s00423-011-0739-1.

6. Vagefi PA, Razo O, Deshpande V, McGrath DJ, Lauwers GY, Thayer SP, et al. Evolving patterns in the detection and outcomes of pancreatic neuroendocrine neoplasms: the Massachusetts General Hospital experience from 1977 to 2005. Arch Surg. 2007;142(4):347-354.

7. Falconi M, Bartsch DK, Eriksson B, Kloppel G, Lopes JM, O'Connor JM, et al. ENETS Consensus Guidelines for the management of patients with digestive neuroendocrine neoplasms of the digestive system: well-differentiated pancreatic non-functioning tumors. Neuroendocrinology. 2012;95(2):120-134. doi: 10.1159/000335587.

8. Anlauf M, Gerlach P, Schott M, Raffel A, Krausch M, Knoefel WT, et al. Pathology of neuroendocrine neoplasms. Chirurg. 2011;82(7):567573. doi: 10.1007/s00104-011-2067-y.

9. Yao JC, Hassan M, Phan A, Dagohoy C, Leary C, Mares JE, et al. One hundred years after "carcinoid": epidemiology of and prognostic factors for neuroendocrine tumors in 35,825 cases in the United States. J Clin Oncol. 2008;26(18):3063-3072. doi: 10.1200/ JC0.2007.15.4377.

10. Ekeblad S, Skogseid B, Dunder K, Oberg K, Eriksson B. Prognostic factors and survival in 324 patients with pancreatic endocrine tumor treated at a single institution. Clin Cancer Res. 2008;14(23):77987803. doi: 10.1158/1078-0432.CCR-08-0734.

11. Ramage JK, Davies AH, Ardill J, Bax N, Caplin M, Grossman A, et al. Guidelines for the management of gastroenteropancreatic neuroendocrine (including carcinoid) tumours. Gut. 2005;54(Suppl 4 iv):1-16.

12. Moller JE, Connolly HM, Rubin J, Seward JB, Modesto K, Pellikka PA Factors associated with progression of carcinoid heart disease. N Engl J Med. 2003;348(11):1005-1015.

13. Palaniswamy C, Frishman WH \& Aronow WS. Carcinoid heart disease. Cardiol Rev 201220 167-176.

14. Akerstrom G, Falconi M, Kianmanesh R, Ruszniewski P, Plockinger U. ENETS Consensus Guidelines for the Standards of Care in
Neuroendocrine Tumors: pre- and perioperative therapy in patients with neuroendocrine tumors. Neuroendocrinology. 2009;90(2):203208. doi: $10.1159 / 000225949$.

15. Pavel M, Baudin E, Couvelard A, Krenning E, Oberg K, Steinmuller T, et al. ENETS Consensus Guidelines for the management of patients with liver and other distant metastases from neuroendocrine neoplasms of foregut, midgut, hindgut, and unknown primary. Neuroendocrinology. 2012;95(2):157-176. doi: 10.1159/000335597.

16. Toste PA, Kadera BE, Tatishchev SF, Dawson DW, Clerkin BM, Muthusamy R, et al. Nonfunctional Pancreatic Neuroendocrine Tumors $<2 \mathrm{~cm}$ on Preoperative Imaging are Associated with a Low Incidence of Nodal Metastasis and an Excellent Overall Survival. J Gastrointest Surg. 2013;17(12):2105-2113. doi: 10.1007/s11605013-2360-9.

17. Sharpe SM, In H, Winchester DJ, Talamonti MS, Baker MS. Surgical resection provides an overall survival benefit for patients with small pancreatic neuroendocrine tumors. J Gastrointest Surg. 2015;19(1):117-123. doi: 10.1007/s11605-014-2615-0.

18. Hasegawa T, Yamao K, Hijioka S, Bhatia V, Mizuno N, Hara K, et al. Evaluation of Ki-67 index in EUS-FNA specimens for the assessment of malignancy risk in pancreatic neuroendocrine tumors. Endoscopy. 2014;46(1): 32-38. doi: 10.1055/s-0033-1344958.

19. Hashim YM, Trinkaus KM, Linehan DC, Strasberg SS, Fields RC, Cao D, et al. Regional Lymphadenectomy Is Indicated in the Surgical Treatment of Pancreatic Neuroendocrine Tumors (PNETs). Ann Surg. 2014;259(2):197-203 doi: 10.1097/SLA.0000000000000348.

20. Curran T, Pockaj BA, Gray RJ, Halfdanarson TR, Wasif N. Importance of lymph node involvement in pancreatic neuroendocrine tumors: impact on survival and implications for surgical resection. J Gastrointest Surg. 2015;19(1):152-160; discussion 160. doi: 10.1007/ s11605-014-2624-z.

21.Jilesen AP, Busch OR, van Gulik TM, Gouma DJ, Nieveen van Dijkum EJ. Standard pre- and postoperative determination of chromogranin a in resectable non-functioning pancreatic neuroendocrine tumors-diagnostic accuracy: NF-pNET and low tumor burden. Dig Surg. 2014;31:407-414. doi: 10.1159/000370007.

22. Keutgen XM, Nilubol N, Glanville J, Sadowski SM, Liewehr DJ, Venzon DJ, et al. Resection of primary tumor site is associated with prolonged survival in metastatic nonfunctioning pancreatic neuroendocrine tumors. Surgery. 2016;159(1):311-319. doi: 10.1016/j. surg.2015.05.042.

23. Capdevila J \& Tabernero J. A shining light in the darkness for the treatment of pancreatic neuroendocrine tumors. Cancer Discov. 2011;1(3):213-221. doi: 10.1158/2159-8290.CD-11-0151.

24. Modlin IM, Oberg K, Chung DC, Jensen RT, de Herder WW, Thakker RV, et al. Gastroenteropancreatic neuroendocrine tumours. Lancet Oncol. 2008;9(1):61-72. doi: 10.1016/S1470-2045(07)70410-2.

25. Jablonska B, Dranka-Bojarowska D, Palacz H, Lewinski A \& Lampe P. Surgical treatment of pancreatic neuroendocrine tumours - clinical experience. Pol Przegl Chir. 2011;83(4):216-222. doi: 10.2478/ v10035-011-0033-5. 